\title{
Le rôle des lidars dans l'étude de l'atmosphère
}

\author{
M.-L. Chanin
}

Service d'Aéronomie du CNRS, BP. 3, 91370 Verrières-le-Buisson, France

\section{RÉSUMÉ}

L'utilisation des lidars pour l'étude de l'atmosphère a connu un grand développement au cours des deux dernières décennies. La possibilité de sonder à distance et sans perturbation du milieu les paramètres physiques, tels que la densité, la température, le vent, la turbulence, ainsi que la concentration d'un certain nombre de constituants de l'atmosphère a ouvert leur domaine d'application à l'étude de l'environnement qu'il soit proche ou lointain. Nous décrirons plus particulièrement quelques types de lidars développés en France et utilisés tant pour la recherche que pour des applications civiles et militaires et évoquerons leur utilisation future à partir de plates-formes spatiales.

\section{INTRODUCTION}

Le sondage de l'atmosphère a connu un développement spectaculaire, parallèlement à l'augmentation des performances des lasers eux-mêmes : l'énergie par pulse, le taux de répétition, la monochromaticité, le domaine spectral accessible et dans tous les cas la fiabilité. Les modes d'interaction du faisceau laser avec l'atmosphère pouvant être utilisés sont variés et dépendent du paramètre que l'on cherche à mesurer et de la portée recherchée. Nous rappellerons brièvement le principe du lidar et ses avantages avant de décrire plus précisément quelques types d'application de ceux-ci et quelques exemples de résuitats plus particulièrement à partir de lidars développés en France.

\section{GÉNÉRALITÉS}

\subsection{Principe général}

Le principe du lidar est assez voisin de celui du radar, si ce n'est que l'onde envoyée dans l'atmosphère se situe dans le domaine optique, d'où le nom de LIDAR, acronyme de "LIght Detection And Ranging" qui a été donné à la méthode. Un lidar comporte toujours un émetteur et un récepteur. L'émetteur est un laser pulsé émettant dans un domaine de transparence de l'atmosphère soit entre approximativement 0,3 et 10 microns. Le choix de la longueur d'onde 
dépend du paramètre ou du constituant à mesurer et du mode d'interaction lumière-matière sélectionné. Après interaction avec l'atmosphère, les photons successivement rétrodiffusés par les couches atmosphériques sont collectés par un télescope (ou un ensemble de ceux-ci) et l'analyse temporelle du signal reçu restitue la répartition spatiale du paramètre ou du constituant mesuré. La durée du pulse, variable entre quelques dizaines de nanosecondes et la microseconde, permet des résolutions spatiales de 1 à 150 mètres. Un cas particulier pour lequel ceci ne s'applique pas correspond à l'utilisation de cibles pour réfléchir le faisceau, option qui est couramment utilisée pour des mesures de pollution locale.

Parmi les processus d'interaction pouvant donner lieu à la rétrodiffusion du faisceau laser, les plus utilisés par les lidars sont la diffusion Rayleigh par l'ensemble des molécules, la diffusion Mie par les particules (aérosols, nuages, poussières), et la diffusion Raman permettant d'isoler des molécules spécifiques. Ces trois modes de diffusion existent quelle que soit la longueur d'onde utilisée avec cependant des efficacités très variables qui en limitent l'application. Un des processus les plus efficaces, la diffusion résonnante qui nécessite des longueurs d'onde spécifiques, a été l'un des plus utilisés à l'origine, notamment en France pour la détection d'espèces atomiques d'origine météoritique présentes dans la haute atmosphère $(\mathrm{Na}, \mathrm{K}, \mathrm{Li}, \mathrm{Fe}, \mathrm{Ca})$, mais ces mesures sont actuellement interrompues en France. Le tableau 1 donne des ordres de grandeur de l'efficacité des différents processus caractérisés chacun par la valeur de la section efficace.

\section{PROCESSUS}

Diffusion élastique

- Rayleigh

- Mie

Diffusion résonnante

Fluorescence

Diffusion Raman

Diffusion Raman résonnante

\section{CARACTERISTIQUES}

1 réception $=1$ émission

$\sigma \sim 10^{-28} \mathrm{~cm}^{2}\left(1^{-4}\right)$

$\sigma \sim 10^{-10} \mathrm{~cm}^{2}$

1 réception $=1$ émission

$\sigma \sim 10^{-12} \mathrm{~cm}^{2}$ (atomes)

$\sigma \sim 10^{-17} \mathrm{~cm}^{2}$ (molécules)

1 réception $\neq 1$ émission

$\sigma \sim 10^{-18} \mathrm{~cm}^{2}$

1 réception $\neq 1$ émission

$\sigma \sim 10^{-29} \mathrm{~cm}^{2}$

1 réception $\neq 1$ émission

$\sigma \sim 10^{-25} \mathrm{~cm}^{2}$

Tableau 1 : Processus de diffusion applicables au sondage par lidar des paramètres atmosphériques.

L'équation simplifiée du lidar s'écrit :

$$
\mathrm{P}(\mathrm{r}, \mathrm{r}+\mathrm{dr})=\mathrm{P} 0 \cdot \mathrm{G}(\mathrm{r}) \cdot \tau_{\mathrm{n}}(\mathrm{r}, \mathrm{r}+\mathrm{dr}) \cdot \exp \left[-2 \tau_{\mathrm{a}}(\mathrm{o}, \mathrm{r}+\mathrm{dr})\right]
$$

où $\mathrm{P}$ représente la puissance moyenne reçue à partir de l'élément diffuseur à la distance comprise entre $r$ et $r+d r$, et $P_{0}$ la puissance moyenne émise par le laser.

$\mathrm{G}(\mathrm{r})$ est un facteur lié à la géométrie du système qui varie en $\mathrm{A} \cdot \mathrm{r}^{-2}$, si A est la dimension de la surface collectrice, et inclut le rendement des différents composants optiques ou photoélectriques du système.

$\tau_{\mathrm{n}}(\mathrm{r}, \mathrm{r}+\mathrm{dr})$ représente l'épaisseur optique de l'élément diffusant à la longueur d'onde d'émission et s'écrit : $\tau_{\mathrm{n}}=\Sigma_{\mathrm{i}}\left(\mathrm{n}_{\mathrm{i}} \mathrm{s}_{\mathrm{i}}\right) \mathrm{dr}$, pour un constituant $\mathrm{i}$ de concentration $\mathrm{n}_{\mathrm{i}}$, et de section efficace de diffusion $\mathrm{si}$. 
$\tau_{\mathrm{a}}(0, \mathrm{r}+\mathrm{dr})$ représente l'épaisseur optique des éléments absorbants, entre le sol et la zone diffusante et s'exprime de la même façon $\tau_{\mathrm{a}}=\sum_{\mathrm{j}} \mathrm{n}_{\mathrm{j}} \mathrm{sj}_{\mathrm{j}}$.

Deux modes d'application de la lumière rétrodiffusée peuvent être utilisés: soit la détermination de la densité est obtenue à partir de l'intensité (et éventuellement de la répartition spectrale) de la rétrodiffusion, soit elle est obtenue à partir de l'absorption de la lumière retrodiffusée par le constituant à mesurer, méthode dite d'absorption différentielle, ou DIAL, qui nécessite deux longueurs d'onde, l'une se situant dans une bande d'absorption et l'autre en dehors de celle-ci.

A partir de l'équation ci-dessus, on voit facilement que pour un lidar donné dont la "Puissance" est définie par le produit de la puissance moyenne du laser P0 par la dimension de la surface collectrice $\mathrm{A}$, la possibilité de mesurer un constituant ou les grandeurs physiques qui peuvent en être déduites, dépend de l'efficacité de l'interaction mise en jeu, de l'abondance du constituant à l'altitude de la mesure et de la distance au récepteur du point de mesure, $r$, ainsi que de la résolution spatiale, dr, choisie. Toute mesure par lidar a donc une portée limite liée à la "Puissance" de celui-ci.

La puissance d'un lidar est usuellement définie par le produit Po.A et s'exprime en W. $\mathrm{m}^{2}$. Pour atteindre une grande portée et une précision satisfaisante, il conviendra donc d'augmenter ce produit : on choisira un laser de grande puissance moyenne, celle-ci étant proportionnelle au produit du nombre de photons/pulse par le taux de répétition. A même puissance moyenne on choisira le laser fournissant le plus grand nombre de photons/pulse si on souhaite travailler de jour pour améliorer le rapport signal sur bruit. En ce qui concerne I'augmentation de la surface collectrice $\mathrm{A}$, la tendance a été jusqu'à récemment d'utiliser des télescopes de plus en plus grands quitte à utiliser des mosaïques de miroirs dont le rendement laissait à désirer. Nous venons de montrer dans notre équipe l'intérêt d'utiliser des montages de plusieurs télescopes identiques, notamment avec des miroirs réalisés par le repliquage d'un miroir matrice. Cette possibilité a pu être utilisée grâce au transport de photons collectés au foyer par fibres optiques jusqu'au boîtier de réception et ceci sans perte d'énergie notable (figure 1).

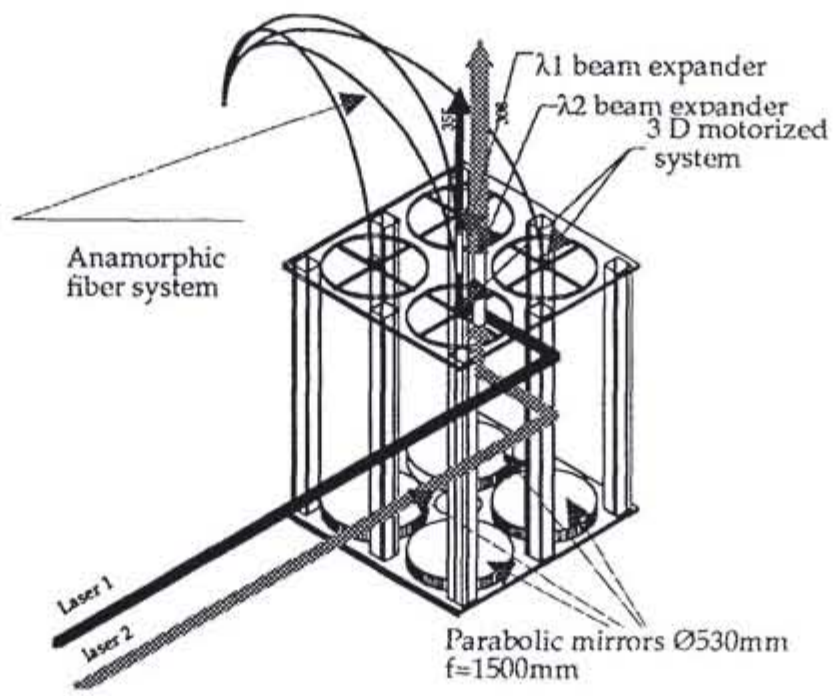

Figure 1: Exemple de récepteurs pour lidars utilisant plusieurs télescopes et une collecte du signal par fibre optique 


\subsection{Les avantages du sondage par lidar}

Le sondage par lidar présente un certain nombre d'avantages liés aux qualités de cohérence spatiale et temporelle des lasers. En effet la faible divergence des lasers permet une bonne localisation de la zone étudiée et autorise l'utilisation de récepteurs de faible ouverture et ainsi une meilleure élimination de la contribution du fond continu, ce qui permet fréquemment des mesures en plein jour. Les courtes durées d'impulsion fournissent une résolution spatiale inaccessible aux autres mesures à distance. La distance au point mesuré est connue de façon absolue avec une grande précision. De plus les qualités spectrales des lasers disponibles aujourd'hui permettent de bénéficier à distance des avantages de la spectroscopie à haute résolution et de disposer localement d'une haute densité spectrale.

Outre ces atouts liés aux qualités des lasers, l'utilisation des lidars offre comme celle des radars la possibilité de mesures continues, c'est-à-dire de surveillance opérationnelle notamment pour la mesure de paramètres qui jusqu'alors n'étaient mesurés que de façon sporadique par ballons et fusées. Enfin les résolutions spatiales et temporelles font des mesures par lidar un complément indispensable des mesures satellitaires.

\section{LA MESURE DES PARAMÈTRES PHYSIQUES}

\subsection{La densité et la température.}

La rétrodiffusion par les molécules de l'air en l'absence de diffusion Mie donne accès à la densité relative de l'atmosphère. La méthode du lidar Rayleigh est donc applicable de $30 \mathrm{~km}$ jusqu'à 90-100 km, cette limite supérieure dépendant de la "Puissance" du lidar. L'ignorance de la transmission de la basse atmosphère, paramètre qui de plus est très variable, ne permet pas d'accéder à la mesure de la densité absolue, si ce n'est par normalisation avec un radiosondage ou un modèle. Un schéma simplifié de lidar Rayleigh est présenté sur la figure 2.

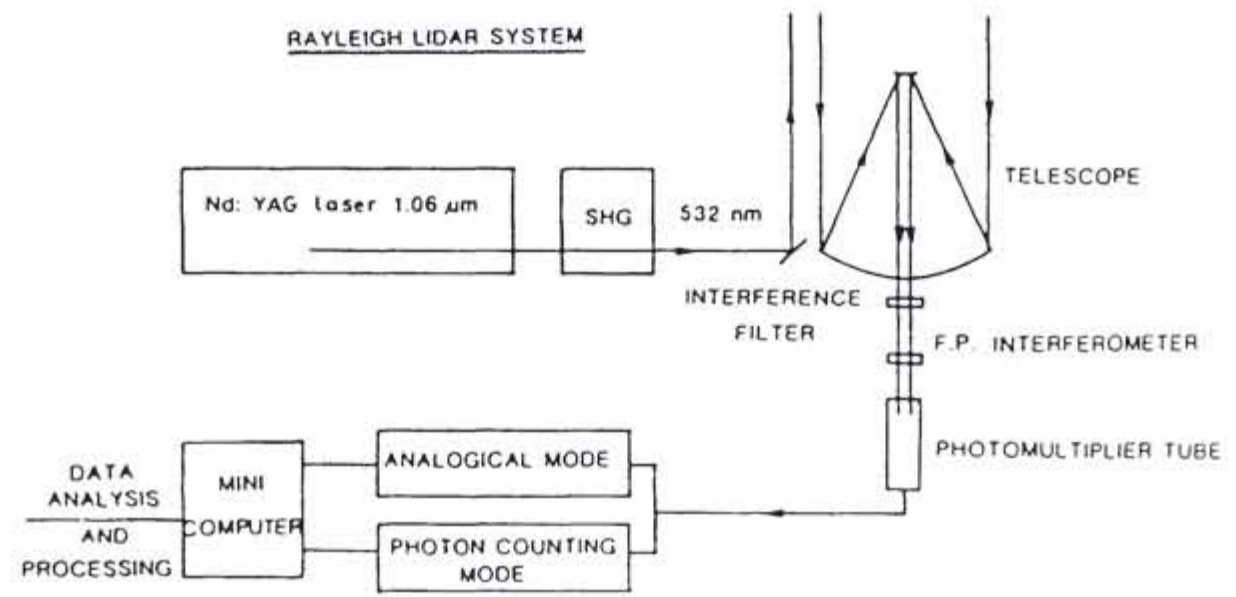

Figure 2 : Schéma type de lidar Rayleigh

La température est calculée à partir de la densité relative en considérant que l'atmosphère se comporte comme un gaz parfait et en se placant dans l'hypothèse de l'équilibre hydrostatique. Ces hypothèses sont satisfaites aux échelles temporelles et spatiales des phénomènes que nous 
étudions. Hormis les $15 \mathrm{~km}$ en haut du profil où la température dépend de la valeur choisie pour initier le calcul, la mesure de la température est absolue et indépendante de la normalisation de la densité (Hauchecorne, and Chanin, 1980.- Chanin, and Hauchecorne,1984.) (figure 3).
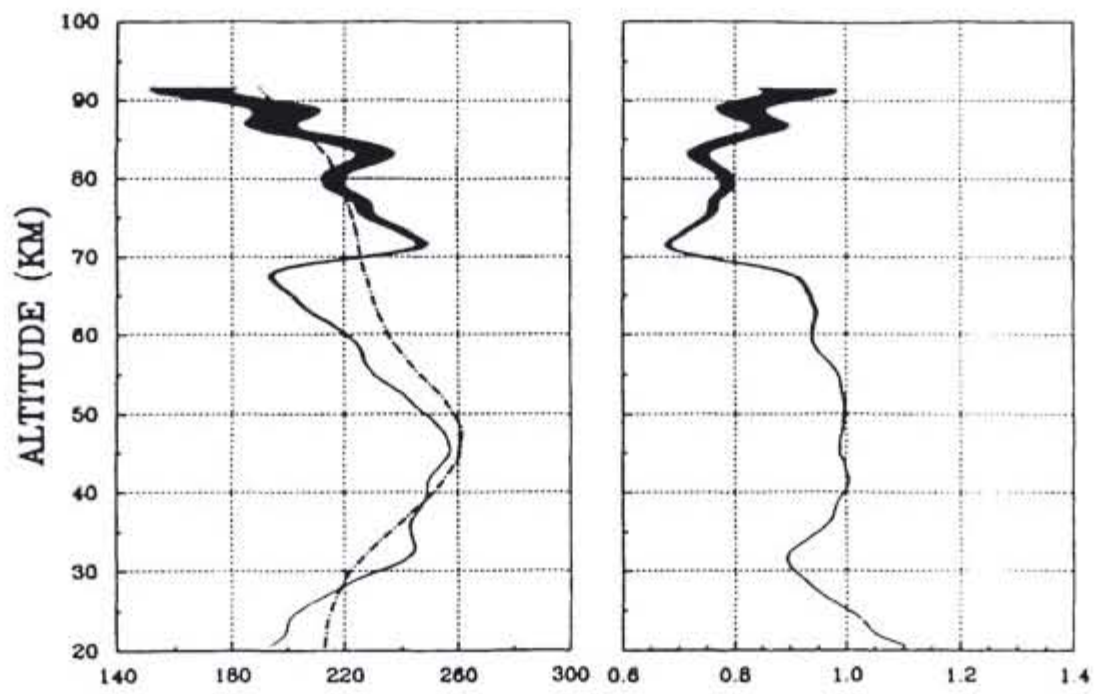

Figure 3 : Profil de température (à gauche) comparé au modèle CIRA 86 (--) et profil de densité normalisé par rapport au modèle, obtenus par lidar Rayleigh au CEL le 2 février 1988.

Le premier instrument de ce type a été développé à l'Observatoire de Haute Provence (OHP) dès 1978 où une version opérationnelle fontionnant en mode semi-automatique date de 1981 . Nous disposons donc à ce jour d'une banque de données de plus de 1200 nuits de mesure couvrant plus d'un cycle solaire. Des lidars similaires ont été installés en 1986 au Centre d'Essais des Landes (CEL) et en 1989 sur le bateau BEM Henri Poincaré, transféré depuis 1992 sur le Monge où il est utilisé de façon opérationnelle. Ce type de lidars est amené à remplacer les fusées qui jusqu'alors assuraient la surveillance de la haute atmosphère.

La base de données de l'OHP complétée par celle du CEL et les mesures faites en mer ont conduit à un grand nombre de résultats sur la variabilité de l'atmosphère à différentes échelles (ondes de gravité, marée, ondes planétaires, influence des cycles solaires, variation d'origine anthrogénique) et les données servent de validation pour les mesures satellitaires et de base pour les modèles.

La présence de diffusion Mie introduit une erreur inacceptable sur la mesure de la densité en dessous de $30 \mathrm{~km}$; c'est la raison pour laquelle nous avons développé le lidar Raman. On peut utiliser au choix les raies vibrationnelles très éloignées spectralement de la raie d'excitation qui ne sont pas perturbées par la diffusion Mie; la mesure de la concentration d'un gaz majoritaire, $\mathrm{N}_{2}$ par exemple, donne alors accès à la densité, puis à la température de la même façon que précédemment (Keckhut et al., 1990). Cette méthode est difficilement utilisable lorsque la contribution de la diffusion Mie est importante, car celle-ci varie en fonction de la longueur d'onde d'une façon dépendante de la nature des aérosols. On préfère alors utiliser les raies Raman rotationnelles de $\mathrm{N}_{2}$ et $\mathrm{O}_{2}$, beaucoup plus proches, et donc affectées de la même façon par l'extinction due à la présence d'aérosols. Dans ce cas, l'enveloppe des raies étant sensible à la température, on a accès directement à ce paramètre sans que soit nécessaire la connaissance de la densité (fig.4). Cette méthode permet depuis peu de compléter le profil de température obtenue par le lidar Rayleigh entre environ 3 et 30 km ( fig.5), (Nedeljkovic et al., 1993). 


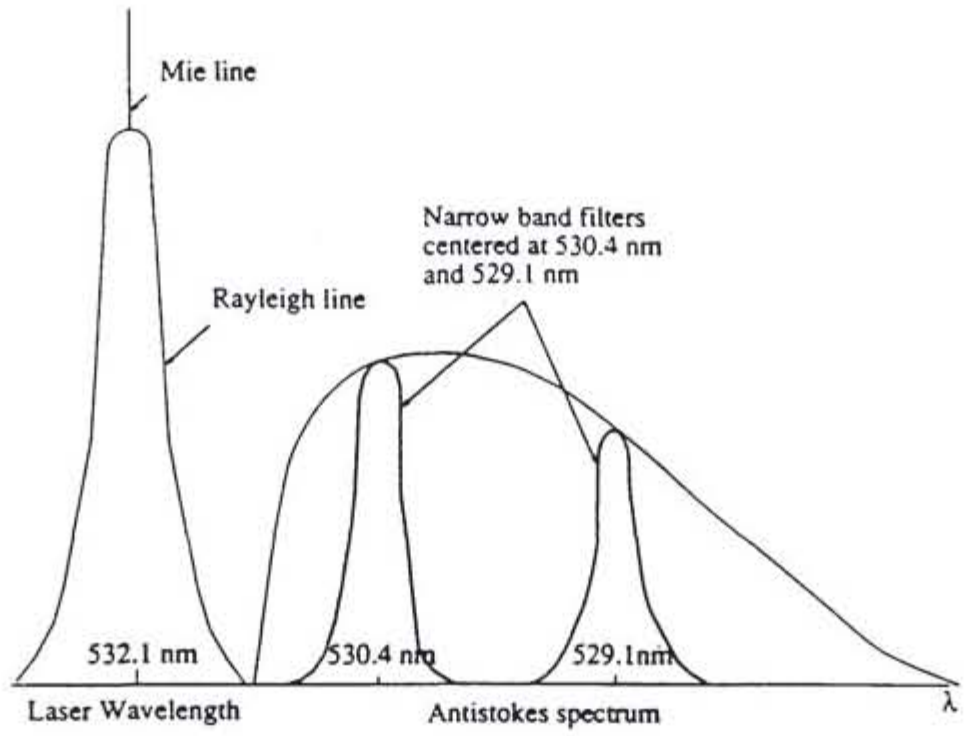

Figure 4 : Principe de la mesure de température utilisant la diffusion Raman rotationnelle

\subsection{Le vent}

La connaissance des composantes du vent est si fondamentale pour la prévision météorologique que les efforts pour mettre au point une méthode de mesure du vent par lidar ont été nombreux. Deux types de lidar Doppler ont été développés, qui l'un et l'autre mesurent le déplacement Doppler dans la direction d'observation : l'un utilise la rétrodiffusion Mie et la détection cohérente, l'autre la diffusion Rayleigh et la diffusion incohérente et nous décrirons brièvement les deux méthodes.

La mesure du vent nécessite une haute résolution spectrale telle que celle qu'offre la détection hétérodyne; mais son application implique de travailler dans le domaine des longueurs d'onde infra-rouges. D'où le choix de la diffusion Mie dont la variation spectrale est approximativement en $\lambda-1,5$ et non en $\lambda^{-4}$ comme la diffusion Rayleigh. Les lidars $\mathrm{CO}_{2}$ utilisés dans ce domaine spectral aux environs de $10 \mu \mathrm{m}$ sont fiables et très puissants; des systèmes opérationnels au sol et embarqués en avion existent déjà depuis plusieurs années. Les travaux en cours en France portent surtout sur le développement de nouveaux algorithmes permettant de restituer la fréquence Doppler avec des performances améliorées, tout en réduisant la Puissance du lidar en vue de systèmes embarqués (Zarader et al. 1993).

Pour résoudre le problème dans une atmosphère propre (c'est-à-dire sans aérosols), il est nécessaire de pouvoir mesurer l'effet Doppler sur la rétrodiffusion Rayleigh; c'est l'objectif du lidar Doppler incohérent. Il utilise une méthode interférométrique à deux voies qui est essentiellement sensible au déséquilibre entre les deux voies causé par un décalage Doppler de la raie rétrodiffusée (Figure 6). Il a été démontré récemment, alors que la basse stratosphère était très chargée en aérosols après l'éruption du Mt Pinatubo, que la méthode pouvait être utilisée en présence de poussières, ce qui élargit son domaine d'utilisation de la basse troposphère à environ $60 \mathrm{~km}$. Un modèle prototype est actuellement au CEL, et une version opérationnelle sera implantée en Avril 1993 à l'OHP; sa portée sera de $60 \mathrm{~km}$ comme le montre la figure 8. 


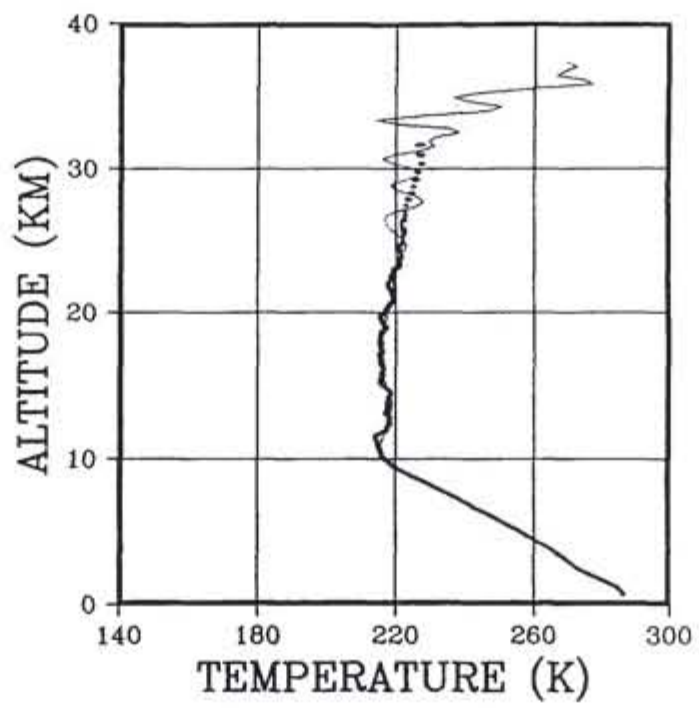

Figure 5 : Profil obtenu par Lidar Raman rotationnel le 9 Avril 1991 à l'OHP (--) et profil de radiosondage obtenu simultanément $(\cdot)$.

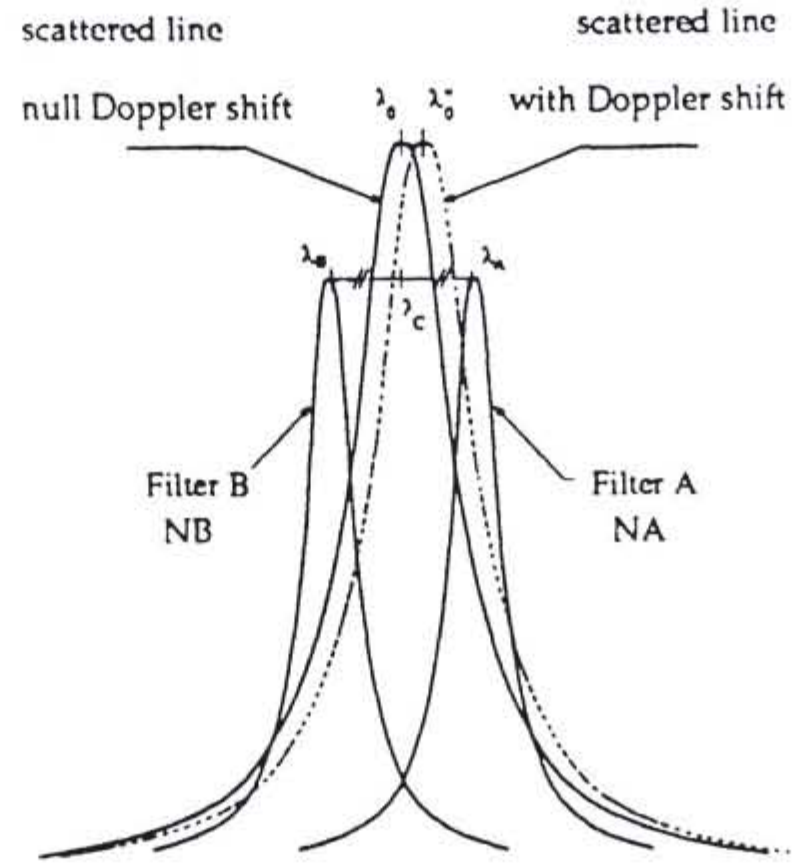

Figure 6 : Principe du Lidar Doppler incohérent imaginé et développé au Service d'Aéronomie 


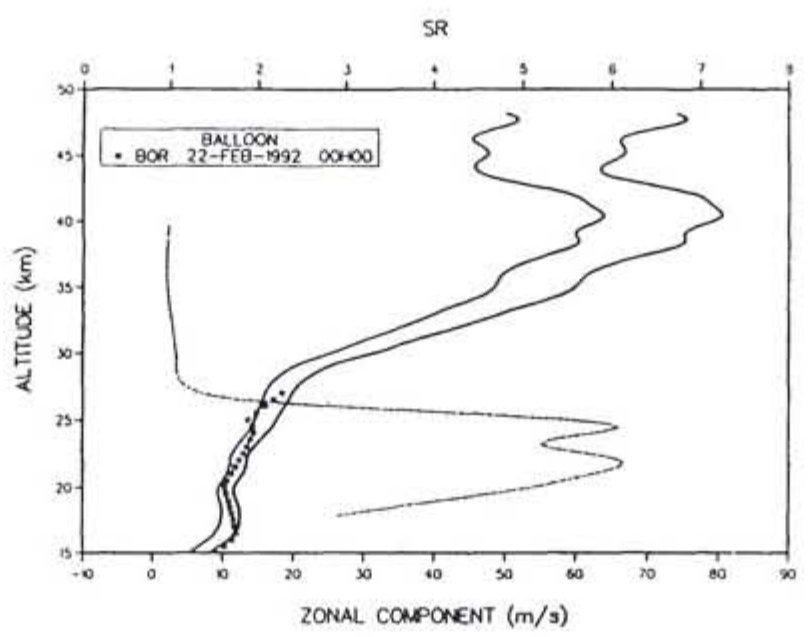

Figure 7 : Profil de vent donné à $+1 \sigma$ par lidar Doppler $\Leftrightarrow$ comparé au profil obtenu par radiosondage (*..). Le profil du rapport de diffusion (en pointillé et échelle supérieurc) montre que la présence des aérosols vers $20-25 \mathrm{~km}$ ne perturbe pas la mesure

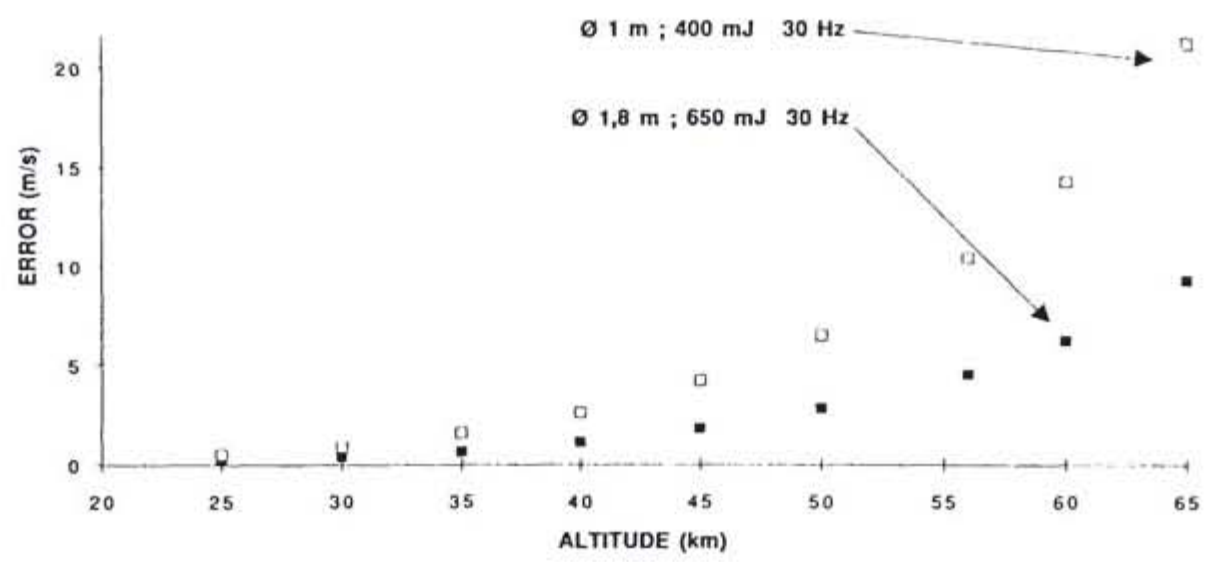

Figure 8 : Erreur statistique pour une mesure de vent effectuée avec le Lidar Doppler incohérent pour une intégration de $30 \mathrm{~min}$. et une résolution verticale de $2 \mathrm{~km}$, (o) pour le lidar Doppler de l'OHP, et (•) pour un lidar Doppler en construction devant être implanté à Andoya (Norvège).

\subsection{La turbulence}

La turbulence atmosphérique induit des fluctuations à petite échelle de la densité, de la température, des trois composantes du vent et de la concentration des aérosols. Bien qu'il ne soit pas possible de mesurer directement les fluctuations induites sur le signal lidar à de si petites échelles, il est possible d'estimer par une approche statistique leur variance et de détecter 
ainsi la présence de couches turbulentes (Hauchecorne, 1992). Une telle détection a déjà été faite en présence de diffusion Mie au niveau de la couche limite planétaire comme cela sera présenté ultérieurement (figure 11). De plus si la mesure donne accès directement à un paramètre physique de l'atmosphère par diffusion Rayleigh pure (densité, température ou vent), il est également possible de quantifier l'intensité de la turbulence.

\section{LA MESURE DES CONCENTRATIONS}

\subsection{Les aérosols et les nuages}

Etant donné l'efficacité du processus de diffusion Mie, le lidar Mie est le plus répandu, car dans sa version la plus simple il ne nécessite pas de grands investissements. La difficulté réside cependant dans la séparation entre la contribution Rayleigh et la contribution Mie surtout dans la basse atmosphère où les deux contributions sont toujours présentes et où l'atténuation est très variable et doit être déterminée. Deux méthodes d'inversion mathématiques permettent cependant de restituer les profils verticaux d'atténuation et de rétrodiffusion: les méthodes logarithmiques, qui requièrent une valeur de référence au delà de la zone étudiée, et les méthodes linéaires qui demandent un étalonnage absolu au moyen d'une cible de réflectivité connue ou, si la portée du lidar le permet, en utilisant la diffusion moléculaire. C'est en particulier la méthode utilisée pour la détection des aérosols stratosphériques d'origine volcanique, de composition relativement bien connue, et pour la surveillance desquels il existe un vaste réseau à l'échelle internationale (Avdyushin et al., 1993).

Cependant la section efficace d'extinction dépend de la taille et de la nature des particules; lorsqu'il s'agit de séparer des gouttelettes d'eau de poussières de nature connue, on peut se contenter d'utiliser 2 ou 3 longueurs d'onde, par exemple les différentes harmoniques du laser Nd-Yag. Mais lorsque l'on doit identifier des aérosols plus complexes, il faut au préalable acquérir une bonne connaissance de la signature spectrale des coefficients d'extinction et de rétrodiffusion des espèces susceptibles d'être mesurées, comme le montre la figure 9. L'identification des particules utilise alors un grand nombre de raies laser, par exemple les transitions d'un laser CO2 comme cela est fait au Centre d'Etudes du Bouchet (Adam, 1993.Cunin,1992).

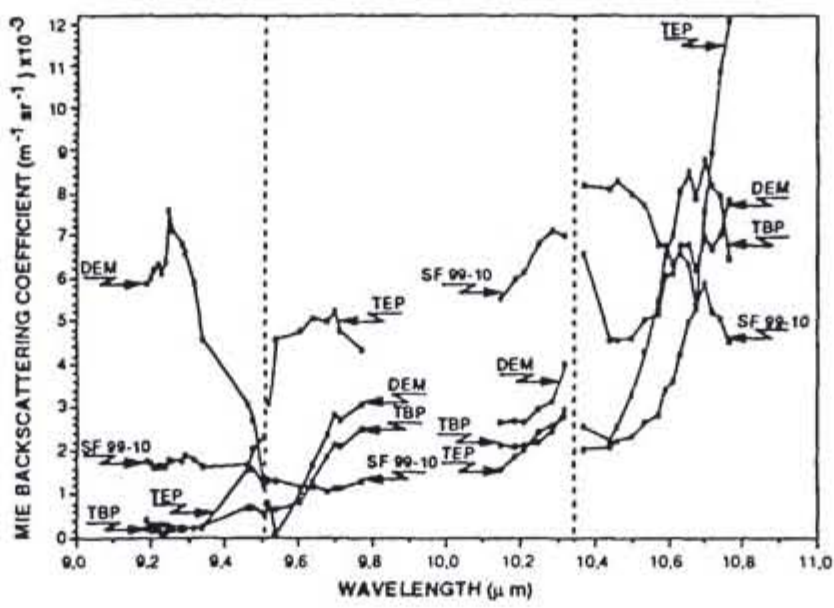

Figure 9 : Coefficient de rétrodiffusion pour un ensemble d'aérosols toxiques pouvant être détectés par rétrodiffusion différentielle (DISC) entre 9 et $11 \mu \mathrm{m}$ (Cunin,1992). 


\subsection{Les constituants en faible rapport de mélange}

A priori le lidar Raman permet d'isoler spectralement tout constituant de l'atmosphère que l'on cherche à mesurer et il est utilisé pour la détection à courte portée de polluants. Lorsque la concentration du constituant est suffisante, la portée peut même permettre d'atteindre la tropopause, ce qui est le cas pour $\mathrm{H}_{2} \mathrm{O}$ (Melfi et al., 1992) et $\mathrm{CH}_{4}$. Mais du fait de la faible efficacité du processus, la méthode la plus utilisée est l'absorption différentielle, utilisant comme support aussi bien la rétrodiffusion Rayleigh que la diffusion Mie, le choix dépendant du domaine d'altitude. C'est notamment la méthode développée en France pour la surveillance de l'ozone stratosphérique et troposphérique, qui fait d'ailleurs partie d'un engagement intemational de surveillance de la stratosphère dans le cadre du NDSC (Network for Detection of Stratospheric Changes).

A titre d'exemple nous décrirons brièvement la méthode DIAL appliquée à la détection de l'ozone; elle utilise l'absorption différentielle et nécessite donc l'émission de deux longueurs d'onde correspondant à une différente section efficace d'absorption par l'ozone (Melfi et al., 1992). La concentration d'ozone est obtenue à partir de la dérivée logarithmique du rapport des signaux reçus, corrigés des termes liés à la diffusion Rayleigh, Mie et à l'absorption par d'autres constituants

Une des difficultés réside dans le choix des paires de longueurs d'onde, de préférence spectralement voisines, mais choisies de façon à obtenir une différence suffisante entre les épaisseurs optiques, tout en optimisant la précision de la mesure. Cela implique de choisir des paires différentes pour les différents domaines d'altitude, et augmente la complexité du lidar. La proximité des deux longueurs d'onde est d'autant plus critique que la concentration en aérosols est élevée. Il s'avère alors indispensable d'utiliser plusieurs lasers : en l'occurrence un laser eximer $\mathrm{XeCl}$ émettant à $308 \mathrm{~nm}$, et un laser Nd-Yag dont on utilise soit le 3 ième harmonique, qui sert de référence pour effectuer la mesure au dessus de $30 \mathrm{~km}$, soit le 4 ième harmonique et des cellules Raman contenant différents gaz pour générer les longueurs d'onde optimisées, 288 et $293 \mathrm{~nm}$ pour la troposphère, et une longueur d'onde proche de $308 \mathrm{~nm}$ pour la basse stratosphère.

Deux lidars, l'un pour la stratosphère, l'autre pour la troposphère fonctionnent à l'OHP, le premier de façon opérationnelle depuis 1986. Ce dernier a permis d'obtenir la variation saisonnière comme le montre la figure 10. L'objectif étant la détection des tendances à long terme, la précision absolue, hormis l'incertitude sur les sections efficaces dont on peut tenir compte a posteriori, doit être de l'ordre de $3 \%$ dans tout le domaine d'altitude.

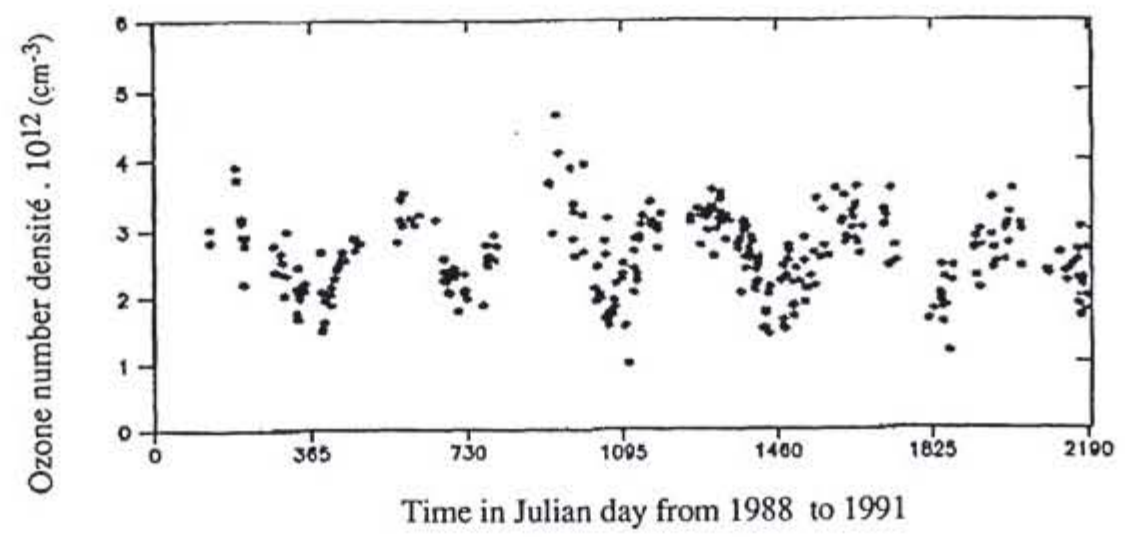

Figure 10: Variation saisonnière de l'ozone stratosphérique à $30 \mathrm{~km}$ à l'OHP entre 1988 et 1991. 


\section{QUELQUES EXEMPLES D'APPLICATION:}

\subsection{La couche limite planétaire}

La couche limite atmosphérique (CLA) est marquée par une forte discontinuité de la rétrodiffusion due à la diminution brutale de poussières ou de la condensation nuageuse au passage dans la troposphère libre. Le calcul de variance sur des profils lidar successifs met en évidence très clairement cette zone de transition, notamment par la présence d'une couche turbulente, alors qu'une inversion logarithmique permet de rétablir un profil de rétrodiffusion absolue comme le montre la figure 11. Le lidar permet donc de mesurer la hauteur de la CLA par le pic de variance et la zone d'entrainement de la CLA par la largeur de ce pic.

Les premières études de la CLA dans plusieurs types de situation ont pu être menées par une équipe du LMD et du SA d'abord au sol, puis grâce au lidar LEANDRE embarqué sur l'avion ARAT. Elle a permis de documenter l'évolution spatiale de la CLA à l'interface terre-mer, en terrain complexe, en présence de relief montagneux et de nuages. L'objectif est l'étude systématique des caractéristiques importantes de cette couche: hauteur de la CLA, zone d'entraînement, turbulence, notamment pour étudier les échanges entre la basse troposphère et la troposphère libre et le couplage dynamique-rayonnement (Pelon et al., 1993, Dupont, 1991).
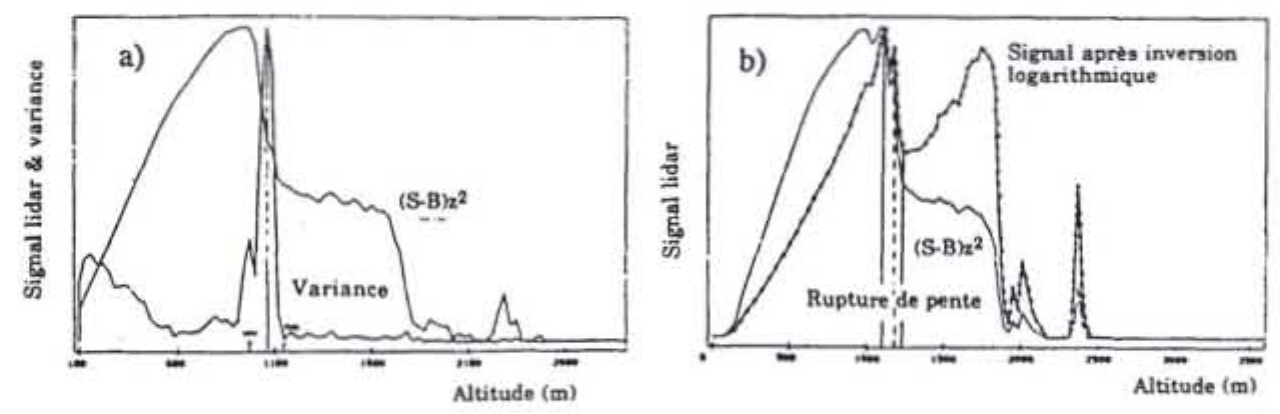

Figure 11 : Détermination de la hauteur de la CLA terrestre et de la zone d'entrainement par : a) la méthode de variance, b) la méthode de rupture de pente après inversion de l'équation lidar par la méthode logarithmique pour restituer le profil de rétrodiffusion (Dupont, 1991).

\subsection{Les échanges troposphère-stratosphère.}

Pour établir les bilans de l'ozone tant dans la stratosphère que dans la troposphère, il est essentiel de chiffrer à l'échelle globale les échanges d'ozone entre les deux régions. La question est loin d'être résolue: on sait que les échanges dans le sens troposphère-stratosphère se produisent majoritairement en région tropicale et que la situation est inversée aux hautes latitudes et dans les deux sens aux latitudes moyennes. On ignore par contre les flux mis en jeu. Un programme d'étude des différentes situations à l'origine de ces transferts d'ozone est en cours à l'OHP. Deux mécanismes ont été mis en évidence lors de plusieurs campagnes menées depuis 1988 : le transfert par foliation de tropopause et le mélange turbulent associé aux gouttes froides. Dans les différents cas les flux ont été évalués et sont de l'ordre de $10^{12} \mathrm{~mol} . \mathrm{cm}^{-2} \mathrm{~s}^{-1}$, 
et l'extension horizontale des événements estimée à un facteur 2 près à $10^{6} \mathrm{~km}^{2}$, ce qui correspond à des transferts d'environ 1,2 à $2,510^{33}$ molécules d'ozone (fig.12). La poursuite du programme mené à l'échelle des Communautés Européennes devra estimer la fréquence de ces événements pour effectuer le bilan global. La figure 12 montre un exemple typique d'un transport lors d'une foliation de tropopause.

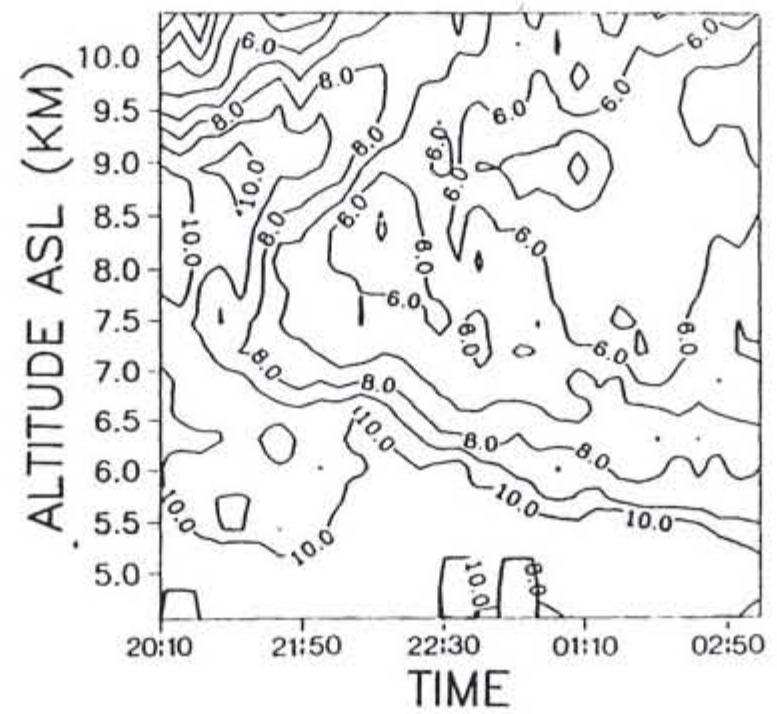

Figure 12 : Evolution temporelle du profil vertical d'ozone mesuré par lidar à l'OHP dans la nuit du 27-28 Mars 1988. Les lignes iso-concentration sont représentées en $10^{11} \mathrm{~mol} / \mathrm{cm}^{3}$.

\subsection{Climatologie de la stratosphère}

La grande base de mesures de température obtenue grâce aux lidars Rayleigh implantés dans le Sud de la France a permis une étude exhaustive des valeurs moyennes et de variabilité de la température entre 30 et $90 \mathrm{~km}$. L'étude porte soit sur des phénomènes de courte période : marées (Gille et al., 1991), ondes de gravité (Wilson, ot al., 1990), ondes planétaires (Hauchecorne and Chanin, 1980), sur l'évolution saisonnière et sur des phénomènes de plus grande amplitude, notamment l'influence du cycle solaire de 11 ans et limpact des changements de composition de l'atmosphère $\left(\mathrm{CO}_{2}, \mathrm{O}_{3}\right)$; Du fait que le lidar Rayleigh couvre un domaine d'altitude qui n'était sondé que de façon sporadique auparavant, un grand nombre des résultats obtenus sont originaux. Ils ont permis de mettre en évidence par exemple l'influence solaire (fig.13 ) qui d'ailleurs est en opposition de phase dans la stratosphère indiquant qu'il s'agit d'un effet indirect. Le réchauffement de la mésosphère (environ $65 \mathrm{~km}$ ) au maximum du cycle solaire s'explique simplement par l'absorption d'un plus grand flux UV en période de maximum d'activité solaire.

Un des résultats les plus important du point de vue climatique est la mise en évidence d'un refroidissement de $4 \mathrm{~K}$ par décennie de la mésosphère (Hauchecorne et al., 1991), (fig.14 ) explicable par l'augmentation du gaz carbonique qui contribue à renvoyer vers l'espace une fraction plus importante du flux solaire incident. L'évolution dans la haute stratosphère (30-50 $\mathrm{km}$ ) est incertaine; dans ce domaine la combinaison de la diminution d'ozone et de l'augmentation du gaz carbonique laisse prévoir un net refroidissement qui n'est pas observé, 
alors qu'un tel effet est clairement identifié à $17-20 \mathrm{~km}$, altitude du maximum de diminution d'ozone en régions polaires (Miller et al.1992).

Response to solar flux (summer 1979-1991)

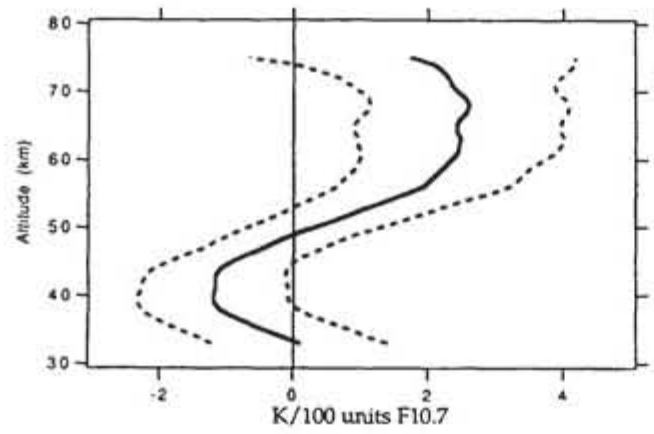

Linear trend (summer 1979-1991)

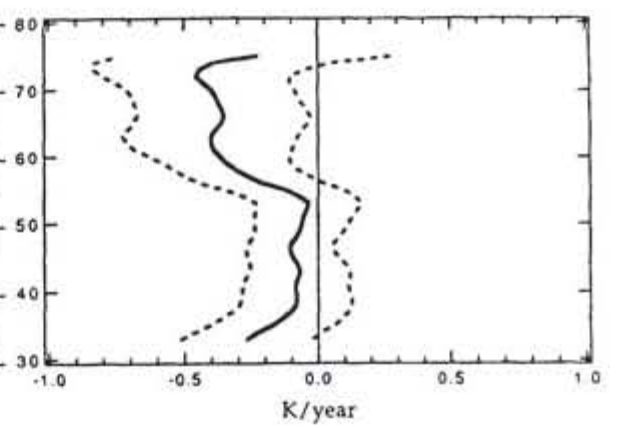

Figure 13 (left) and Figure 14 (right) Response to solar flux and linear trend due to anthropic changes, using the data base of Rayleigh lidar from $\mathrm{OHP}\left(44^{\circ} \mathrm{N}, 6^{\circ} \mathrm{E}\right)$ and $\operatorname{CEL}\left(44^{\circ} \mathrm{N}, 1^{\circ} \mathrm{O}\right)$ from 1979 to 1991.

\subsection{Les inversions mésosphériques}

Les profils de température obtenus par lidar Rayleigh montrent fréquemment une inversion de température dans la mésosphère pouvant atteindre des amplitudes de 30 à $40 \mathrm{~K}$ (voir fig.3). Ces inversions persistent plusieurs jours et leur extension horizontale est de l'ordre de $1000 \mathrm{~km}$. Une étude statistique de ces inversions a été effectuée à partir de plus de 500 profils obtenus depuis 1984; elle a mis en évidence une variation saisonnière de l'altitude où se produit l'inversion et de la fréquence d'occurrence qui laisse penser que leur formation est liée à la turbulence générée par le déferlement des ondes de gravité (Hauchecorne et al., 1991). Cette hypothèse a été d'ailleurs vérifiée par modélisation pendant l'hiver.

Très récemment dans le cadre de la coopération avec différents expérimentateurs du satellite UARS (Upper Atmosphere Research Satellite), nous avons montré que ces inversions existaient à l'échelle globale, avec cependant des régions où leur formation semble se produire de façon préférentielle, et notamment dans la région correspondant à nos sites d'observation en France, ce qui devrait permettre d'élucider la cause de ces inversions.

L'existence de telles couches d'inversion, qui correspondent à des trous de densité de 20 à $40 \%$, est particulièrement critique pour la rentrée dans l'atmosphère d'engins spatiaux, car la zone de freinage se situe vers $70-90 \mathrm{~km}$. Ceci concerne tout particulièrement les avions spatiaux et les missiles.

\subsection{La détection de la pollution et des agents chimiques nocifs}

Le Centre d'Etudes du Bouchet (CEB) a acquis au cours des 15 dernières années une grande expérience dans la détection à distance des éléments polluants et neuro-toxiques qu'ils soient sous forme gazeuse, aérosol ou liquide (Adam, 1993. - Cunin, 1992). Etant donné la complexité des constituants à détecter, le meilleur domaine spectral pour leur détection par analyse spectrale s'avère être l'Infra Rouge. Les lasers utilisés ont donc été des lasers à $\mathrm{CO}_{2}$ et le traitement du signal a fait appel aux techniques de détection directe qui ne permettent que des portées limitées Ultérieurement la technique de détection hétérodyne permettra d'augmenter les 
performances des systèmes et leur utilisation à moyenne portée ( 2 à $5 \mathrm{~km})$, notamment pour les alertes chimiques.

Le principe de détection est le DIAL pour les éléments sous forme gazeuse et le DISC pour les particules, l'un et l'autre décrits plus haut. L'abondance des raies pouvant être émises par les lasers $\mathrm{CO}_{2}$ permet de sélectionner une raie de référence, située hors de toute absorption et d'utiliser les nombreuses raies entre 9 et $11 \mu \mathrm{m}$ pour balayer le domaine spectral où les divers constituants présentent des bandes d'absorption. (fig.15). Le principe peut être mis en oeuvre sur un signal réfléchi sur une cible ou rétrodiffusé par l'atmosphère. L'objectif du CEB est le développement de systèmes opérationnels pouvant être utilisés pour la surveillance de routine: plusieurs générations de tels lidars ont déja vu le jour au CEB et la figure 16 représente le schéma d'une des plus récentes réalisations.

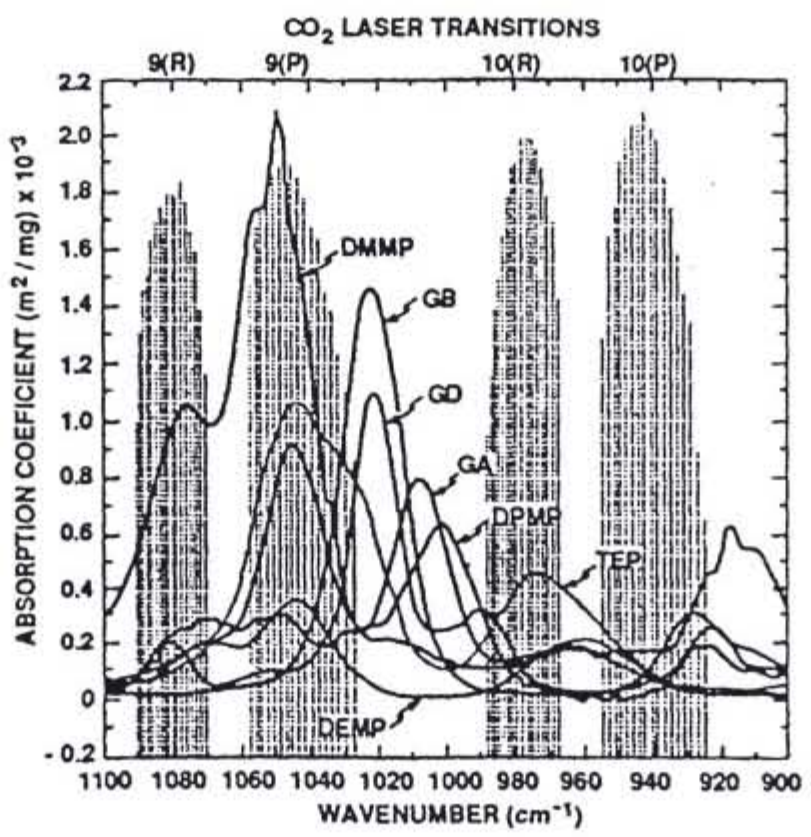

Figure 15 : Coefficients d'absorption mesurés au CEB pour quelques composés organo- phosphatés neurotoxiques (Adam, 1993 - Cunin, 1992).

\section{CONCLUSION}

Les lidars sont amenés à connaître d'importants développements au cours des prochaines années du fait des progrès réalisés dans le domaine des lasers. Ces systèmes vont tendre à s'imposer non seulement comme instruments de recherche mais comme instruments opérationnels dans les domaines de la pollution de l'environnement, de la météorologie et d'une façon générale de la surveillance de l'atmosphère. Deux tendances complémentaires s'imposent : la mise en place de réseaux et le développement de lidars embarqués. 


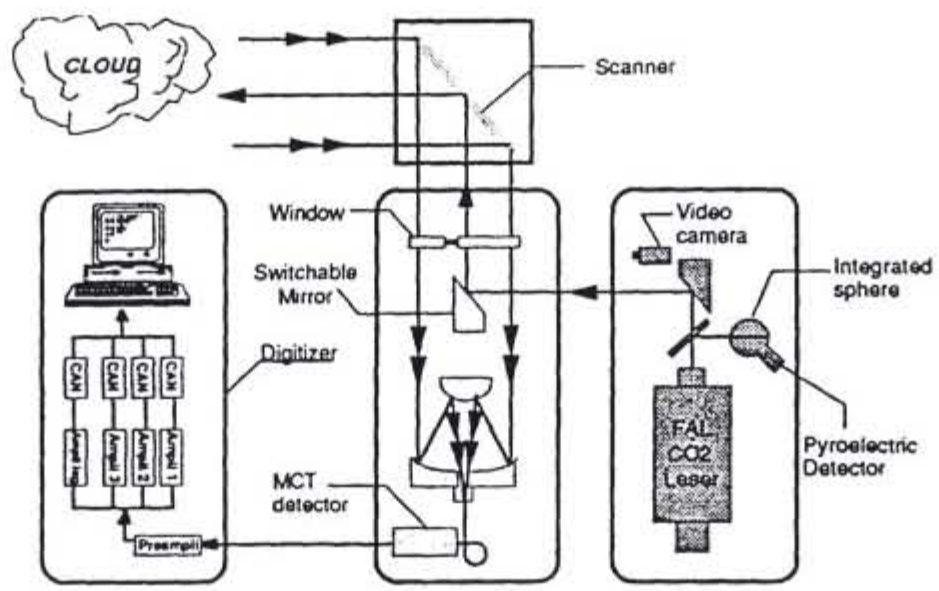

Figure 16: Configuration du nouveau lidar compact MIRELA pour la détection des aérosols (Cunin,1992).

\subsection{Les développements laser}

Les progrès dans le domaine des lasers portent surtout actuellement sur les lasers solides, qu'ils soient utilisés pour le pilotage et l'excitation des lasers actuellement opérationnels, tels que les Nd-Yag, ce qui permet à la fois un gain en finesse et en stabilité spectrale et une plus grande durée de vie, ou qu'ils soient utilisés directement comme source émettrice compacte, de haute cadence, et accordable du visible à l'infrarouge. Ces progrès sont appréciables pour les instruments au sol, tant pour la mesure des paramètres physiques (le vent) que pour la detection de constituants avec une meilleure sensibilité. Mais ils sont essentiels pour les instruments embarqués et notamment pour la mesure du vent.

\subsection{Les réseaux}

Nous avons déjà mentionné que, dans certains domaines, des réseaux se mettaient en place (dans la stratosphère notamment), mais nous ne sommes qu'au début de ce processus. Le rôle des lidars dans la surveillance de l'atmosphère, pour les alertes liées à la pollution ou à la dispersion de gaz toxiques, pour les études de processus atmosphériques, ainsi que pour la validation des mesures satellitaires, n'a été reconnu que récemment.

\subsection{Lidars aéroportés}

L'étude de la stratosphère à partir d'avions instrumentés s'est beaucoup développée, en partie pour comprendre l'impact de l'augmentation du trafic aérien et du vol probable d'une flotte supersonique. Les plus gros porteurs sont alors équipés de lidars pointant au nadir ou au zénith et capables de mesurer les aérosols, la vapeur d'eau, l'ozone, ainsi que le vent. La communauté française dispose du lidar LEANDRE mesurant dans sa première phase les aérosols à partir de l'Avion de Recherche Atmosphérique (A.R.A.T). Elle a l'intention au cours des prochaines années d'équiper dans un cadre européen le prototype d'Airbus 320-01. 


\subsection{Les lidars embarqués sur plate-forme spatiale}

Plusieurs lidars sont à l'étude pour être placés sur les futures plates-formes spatiales. Tout d'abord des lidars destinés à la mesure des nuages et des aérosols : ALISSA réalisé en coopération franco-russe devrait voler en 1994, ATLID dans le cadre européen devrait voir le jour dans les années à venir. Plusieurs projets de lidar Doppler spatiaux font l'objet de collaboration NASA-CNES, mais ils devront sans doute attendre la disponibilité de lasers solides à $2 \mu \mathrm{m}$ plus facilement embarquables que les lasers à $\mathrm{CO}_{2}$ (Lieutaud et al. 1992). Une version aéroportée en collaboration franco-allemande devrait voler fin 1995 (Kopp and Loth, 1993).

\section{Références}

[1] Adam P., Revue Technique Science et Défense sous presse (1993)

[2] Ancellet G., Pelon J., Beekmann M., Papayannis A., Megie G, Proceeding Quadrennial Intern. Ozone Symp., Charlottesville, Virginia, (1993) sous presse

[3] Avdyushin S. I., Tulinov G. F., Ivanov M.S., Kuzmenko B.N., Mezhuev I. R., Nardi B., Hauchecorne A., Chanin M.L., Geophys. Res. Lett. , 20, (1993) 1967-1970

[4] Chanin M.L., Garnier A., Hauchecorne A., Porteneuve J., Geophys. Res. Let., 16, (1989) 1273

[5] Chanin M.L., Hauchecorne A., (MAP Handbook 1984), Vol. 13, pp 87-99

[6] Cunin P., Premier Colloque International sur "les Problèmes de Pollution et d'Environnement", Budapest, octobre 1992

[7] Dupont E., Études méthodologique et expérimentale de la couche limite atmosphérique par télédétection laser, Thèse de Doctorat en Sciences, Univ. P. et M. Curie, 1991.

[8] Gille S.T., Hauchecorne A. and Chanin M.L, J. Geophys. Res., 96, (1991) 7579. 7587

[9] Godin S., Megie G., Pelon J., Geophys. Res. Lett., 16, (1989)

[10] Hauchecorne A. and Chanin M.L., Geophys. Res. Lett. , 7, (1980),565-568.

[11] Hauchecorne A., Chanin M.-L., and Wilson R., Geophys. Res. Lett., 14, (1987) 933936

[12] Hauchecorne A., Chanin M.L., and Kekhut P., J. Geophys. Res. Lett., 96, (1991), 15.297-15.309

[13] Hauchecorne A., Chanin M.L., Geophys.Res.Lett., 7, (1980) 564-568

[14] Hauchecorne A., Communication Privée, 1992.

[15] Keckhut Ph., Chanin M.L., and Hauchecorne A., Applied Optics, 29, (1990) $5182-$ 5186

[16] Köpp F., and Loth C., (WIND Phase-B Mid-T erm Report, Février 1993)

[17] Lieutaud F., Flamant P.H., and Dabas A., Performance of a space borne doppler wind lidar : a parametric analysis at $2 \mathrm{~m}$ and $9.1 \mathrm{~m}$. Final Report ESA Septembre 1992.

[18] Melfi S.H., Whiteman D., Ferrare R., Evans K., Goldsmith J.E.M., Lapp M., and Bisson S.E., Proceeding "16 th Laser Radar Conference", Cambridge, Juillet 1992.

[19] Milles A., Nagatari JRM., Tiao GC., Niu XF., Reinsel GC., Wuebbles D., and Grant K., Geophys. Res. Lett., 19, (1992) 269-272

[20] Nedeljkovic D., Hauchecorne A., and Chanin M.L., IEEE Trans. on Geosciences And Remote Sensing, 31, (1993)

[21] Pelon J., Flamant P.H., Mégie G., and Meissonnier M., Applied Optics, (1993) sous presse

[22] Wilson R., Hauchecorne A., and M.L. Chanin, Geophys. Res. Lett.,, 17, (1990) $1585-1588$

[23] Zarader J.-L., Ancellet G., Dabas A., M'Sirdi N., and Flamant P.H., J. Atmos.Ocean. Techn. (1993) sous presse. 